\title{
Bronchial Infection, CTCAE
}

National Cancer Institute

\section{Source}

National Cancer Institute. Bronchial Infection, CT CAE. NCI Thesaurus. Code C143339.

A disorder characterized by an infectious process involving the bronchi. 